\title{
Reversal of Multidrug Resistance in Mouse Lymphoma Cells by Extracts and Flavonoids from Pistacia integerrima
}

\author{
Abdur Rauf ${ }^{1 *}$, Ghias Uddin², Muslim Raza ${ }^{3}$, Bashir Ahmad ${ }^{4}$, Noor Jehan ${ }^{1}$, Bina \\ S Siddiqui ${ }^{5}$, Joseph Molnar ${ }^{6}$, Akos Csonka ${ }^{6}$, Diana Szabo ${ }^{6}$
}

\begin{abstract}
Phytochemical investigation of Pistacia integerrima has highlighted isolation of two known compounds naringenin (1) and dihydrokaempferol (2). A crude extract and these isolated compounds were here evaluated for their effects on reversion of multidrug resistance (MDR) mediated by P-glycoprotein (P-gp). The multidrug resistance $P$-glycoprotein is a target for chemotherapeutic drugs from cancer cells. In the present study rhodamine123 exclusion screening test on human $\mathrm{mdrl}$ gene transfected mouse gene transfected L5178 and L5178Y mouse T-cell lymphoma cells showed excellent MDR reversing effects in a dose dependent manner. In-silico molecular docking investigations demonstrated a common binding site for Rhodamine123, and compounds naringenin and dihydrokaempferol. Our results showed that the relative docking energies estimated by docking softwares were in satisfactory correlation with the experimental activities. Preliminary interaction profile of P-gp docked complexes were also analysed in order to understand the nature of binding modes of these compounds. Our computational investigation suggested that the compounds interactions with the hydrophobic pocket of P-gp are mainly related to the inhibitory activity. Moreover this study $s$ a platform for the discovery of novel natural compounds from herbal origin, as inhibitor molecules against the P-glycoprotein for the treatment of cancer.
\end{abstract}

Keywords: Pistacia integerrima - naringenin - dihydrokaempferol - multidrug resistance (MDR)

Asian Pac J Cancer Prev, 17 (1), 51-55

\section{Introduction}

Multidrug resistance (MDR) is the main clinical challenge for the active treatment of cancer (chemotherapy) (Szabo and Molnar, 1997). There are numerous mechanisms by which tumor cells develop resistance to antitumor agents. One of them is produced by the overexpression of ATP-binding cassette (ABC) proteins. The ABC transporters represent the largest family of transmembrane proteins that bind ATP and use the energy to drive the transport of various molecules across cell membranes (Gottesman and Ambudkar, 2001; Leonard et al., 2003). ABC efflux transporters extrude a broad range of amphiphilic compounds against the concentration gradient in an energy-dependent fashion. Many of the $\mathrm{ABC}$ transporters have dedicated physiological functions, normal tissue protection in the brain, liver and kidney (Gottesman et al., 2002; Sarkadi et al., 2006; Szakacs et al., 2006).

The first identified drug efflux protein was P-glycoprotein (P-gp, ABCB1) encoded by the $A B C B 1$ gene. $A B C B 1$ is composed of 1280 amino acids $(170 \mathrm{kDa})$ organized in two transmembrane domains (Szakacs et al., 2006). This protein is overexpressed in several human tumors, can extrude wide range of drugs (anticancer, antibiotics, antidepressants, antihistamines, antiarrhythmics, immunosuppressants, HIV protease inhibitors, steroids). Many drug molecules such as tamoxifen, valspodar, dexniguldipine, tariquidar were proposed to suppress the action of $A B C B 1$ (Germann et al., 1993; Lopez and Martinez, 2014) .

Secondly, the Multidrug-Resistant Protein 1 (MRP1, ABCC1) was described in 1992. MRP1 is an efflux pump originally discovered in doxorubicin resistant lung carcinoma cells displaying a multi-drug resistant phenotype without $A B C B 1$ expression (Cole et al., 1992).

MRP1 is expressed ubiquitously at higher level in the blood-brain barrier, intestines and oral mucosa (He et al., 2011). MRP1 expression is higher in the lung than in any other organ and may have protective roles against air pollution and inhaled toxins (Sakamoto et al., 2013). MRP1 physiological substrates include bile acids, folic

${ }^{1}$ Department of Geology, University of Swabi, Ambar, ${ }^{2}$ Institute of Chemical Sciences, ${ }^{4}$ Center of Biotechnology and Microbiology, University of Peshawar, Khyber Pakhtunkhwa, ${ }^{5}$ Institute of Chemistry, International Center for Chemical and Biological Sciences, University of Karachi, Karachi, Pakistan, ${ }^{3}$ State Key Laboratory of Chemical Resource Engineering, Beijing University of Chemical Technology, East Road of North Third Ring, Chao Yang District, Beijing, China, ${ }^{6}$ Department of Medical Microbiology and Immunobiology, Faculty of Medicine, University of Szeged, Szeged, Hungary*For correspondence: mashaljcs@yahoo.com 
acid, leukotriene $\mathrm{C} 4$, glutathione conjugates, and confers resistance to vincristine, methotrexate, doxorubicin, etoposide (Cole and Deeley, 2006). MRP1 expressing lung tumors were identified in $31.6 \%$ and correlated with poor response to cisplatin therapy with vinorelbine, gemcitabine and paclitaxel (Li et al., 2008).

Thirdly, the Breast Cancer Resistance Protein (BCRP) was first cloned by standard protocol (Doyle et al., 1998) in the drug-resistant breast cancer cell line (MCF-7). BCRP is a half-transporter member of the ABCG subfamily (ABCG2) with a size of $72 \mathrm{kDa}$. BCRP probably functions as a homodimer. BCRP expression overlaps largely with $\mathrm{ABCB} 1$, because the protein can be found in tissues like placenta, prostate, small intestine, brain, colon, liver, and ovary (Doyle et al., 1998). Overexpression of BCRP is associated with resistance to a wide range of different anticancer agents including anthracyclines, mitoxantrone, flavopiridol camptothecins and antifolates (Assaraf, 2006; Bihorel et al., 2007; Robey et al., 2007).

Several studies have demonstrated the frequent occurrence of drug efflux proteins in cancer tissue: some authors have reported significant correlations between overexpression of $A B C B 1$ or MRP-1 and poor treatment response in solid tumors and some leukemias (Brinkhuis et al., 2002; Diestra et al., 2003) (Larkin et al., 2004; Damiani et al., 2006), and a prognostic significance for BCRP overexpression in specific forms of leukemia (Larkin et al., 2004).

P-gp belongs to the ABC superfamily of transporters. Members of this family are broadly spread in the three kingdoms and they also play a role in the MDR of pathogen yeasts and bacteria against antimicrobials. Due to its involvement in the treatment of cancer and other human diseases, P-gp is probably the best known of the $\mathrm{ABC}$ proteins and thus, it can be considered as a paradigmatic model for this family of transporters (Jones and George, 2004).

It has 1280 amino acids are arranged as a single chain with two homologous halves having $43 \%$ amino acid identity. A linker region of $\sim 60$ amino acids connects the two halves of the protein. Each half has six transmembrane domains (TM) and a hydrophilic domain containing an ATP-binding site, known as nucleotide binding domain (NBD) (Jara et al., 2013).

The primary binding region for Rhodamine 123 in mouse P-gp was found to be a hydrophobic pocket involving binding site residues such as Ser218, Phe299, Val334, Leu335, Phe339, which have already been proposed to play a crucial role for substrate binding on experimental bases ( $\mathrm{Li}$ et al., 2010).

Pistacia integerrima belongs to family Anacardiacea, commonly known as kakar singhi which is found in Eastern Himalayan range from Indus to Kumaon (Ismail et al., 2011), at height of 12000 to 8000 feet. P. integerrima is a medium sized deciduous tree having medicinal value such as anti-inflammatory, blood purifier, and remedy for gastrointestinal disorders, expectorant, cough, asthma, fever, vomiting and diarrhea according to our former studies (Uddin et al., 2011) (Ahmad et al., 2010). The galls of $P$. integerrima are used as herbal drug for the treatment asthma, diarrhea, chronic bronchitis, disorders of respiratory tract, skin diseases, psoriasis, fever, and as appetizer, hepatitis, liver disorders, oxidative stress and counter hyperuricemia (Uddin et al., 2012a; Uddin et al., 2012b).

Reversal of Multidrug Resistance Mouse Lymphoma cells activity of the extract and its isolated compound has been evaluated which showed remarkable activities.

\section{Materials and Methods}

\section{Assay for reversal of MDR in mouse lymphoma cells}

The L5178 MDR and L5178Y parent cell lines were grown in McCoy`s 5A medium containing $10 \%$ heatinactivated horse serum, was completed with L-glutamine and antibiotics. The cells were adjusted to a density of $2 \times 10^{6} \mathrm{~mL}$ resuspended in serum-free McCoy`s $5 \mathrm{~A}$ medium and distributed in $0.5 \mathrm{~mL}$ aliquots into Eppendorf centrifuge tubes. The tested compound was added at $4 \mu \mathrm{g} /$ $\mathrm{ml}$ final concentrations, and the samples were incubated for 10 minutes at room temperature. Verapamil was applied as positive control (Cornwell et al., 1987) in 10 $\mu \mathrm{g} / \mathrm{ml}$ concentration.

Next, $10 \mu \mathrm{L}$ (5.2 $\mu \mathrm{M}$ final concentration) of the indicator rhodamine 123 (Sigma, St Louis, MO, USA) was added to the samples and the cells were incubated for a further 20 minutes at $37^{\circ} \mathrm{C}$, washed twice and resuspended in $0.5 \mathrm{~mL}$ PBS for analysis. The fluorescence of the cell population was measured with a Partec CyFlow flow cytometer (Münster, Germany). The tested compound was dissolved in DMSO, which was also used as solvent control. The percentage of mean fluorescence intensity was calculated for the treated MDR and parental cell lines as compared with the untreated cells. The activity ratio $\mathrm{R}$ was calculated via the following equation (Cornwell et al., 1987) on the basis of the measured fluorescence values:

$$
\mathrm{FAR}=\frac{\mathrm{MDR}_{\text {treated }} / \mathrm{MDR}_{\text {control }}}{\text { parental }_{\text {treated }} / \text { parental }_{\text {control }}}
$$

\section{Extraction and Isolation}

The shade dried and crushed barks of $P$. integerrima $(14$ $\mathrm{kg}$ ) were defatted with petroleum ether and then subjected to repeated extraction with methanol at room temperature. Then the combined methanol extracts were evaporated under reduced pressure with the aid of a rotary evaporator to afford $600 \mathrm{~g}$ of dried residue. The resulting residue was dissolved in distilled water and sequentially separated with various organic solvents including $n$-hexane, $\mathrm{CHCl} 3$, EtOAc, and n-BuOH. The green residue from the chloroform fraction (15.2 g) was chromatographed on a silica gel column eluted with chloroform/methanol mixtures of increasing polarity $(100: 0 \rightarrow 95: 5, \mathrm{v}: \mathrm{v})$. Fractions obtained were collected and grouped according to their TLC behavior into 10 different sub-fractions (PS-1 to PS-10). Fraction PS-10 obtained with 95:05 chloroform/ methanol was subjected to repeated pencil column chromatography (100:0 $\rightarrow 98: 2)$ to afford a yellowish amorphous solid identified as naringenin (1) followed by a yellow needle crystalline solid, dihydrokaempferol (2). Compounds 1 and 2 were identified by comparing 
their spectral data $\left({ }^{1} \mathrm{H}\right.$ and ${ }^{13} \mathrm{C}$-NMR spectra) with those reported in the literature (Bihorel et al., 2007; Robey et al., 2007). Further elution of the column with $\mathrm{MeOH}-$ chloroform $(0: 100 \rightarrow 3: 97)$ resulted in the isolation of a dark yellow powder which according to TLC consisted of a mixture of two compounds. This mixture was separated into its components by means of preparative TLC to yield dihydrokaempferol (3) and dihydrokaempferol (4); identities of these compounds were confirmed by comparison of their spectral data with those reported

\section{Plant material}

$P$. integerrima (Anacardiacea) were collected in the garden of Sultan Muhammad Razagram, district Dir, K.P.K, Pakistan was recognize by Prof. Dr. A. Rashid of Botany Department University of Peshawar (UOP), Pakistan. A voucher specimen (no, Bot.20037 (PUP) has been deposited in said department.

\section{Computational studies}

The 3-D structure of mice P-glycoprotein (P-gp) was downloaded from protein data bank (PDB) (Berman et al., 2000) with a four letter code of 4Q9L (Resolution $3.80 \AA$ ). Energy refinement of the structure was carried out through Swiss pdb viewer v4.1.0 program implemented with GROMOS96 force field (Guex and Peitsch, 1997). Compound flavonoids structures were prepared for docking studies through Chem sketch (Li et al., 2004) and Avogadro,s software (Hanwell et al., 2012).

Table 1. Docking Interaction Energies of Compounds (1-2) and Rhodamine

\begin{tabular}{lccccc}
\hline Compounds & \multicolumn{2}{l}{ Autodock Vina } & \multicolumn{3}{l}{ i-GEM DOCK } \\
\cline { 2 - 6 } & B. Affinity & Total Energy & VDW & HBond & Elec \\
\hline 1 & -7.5 & -78 & -46 & -32 & 0 \\
2 & -7.3 & -74 & -60 & -14 & 0 \\
Rhodamine123 & -8.2 & -87 & -86 & -1 & 0 \\
\hline
\end{tabular}

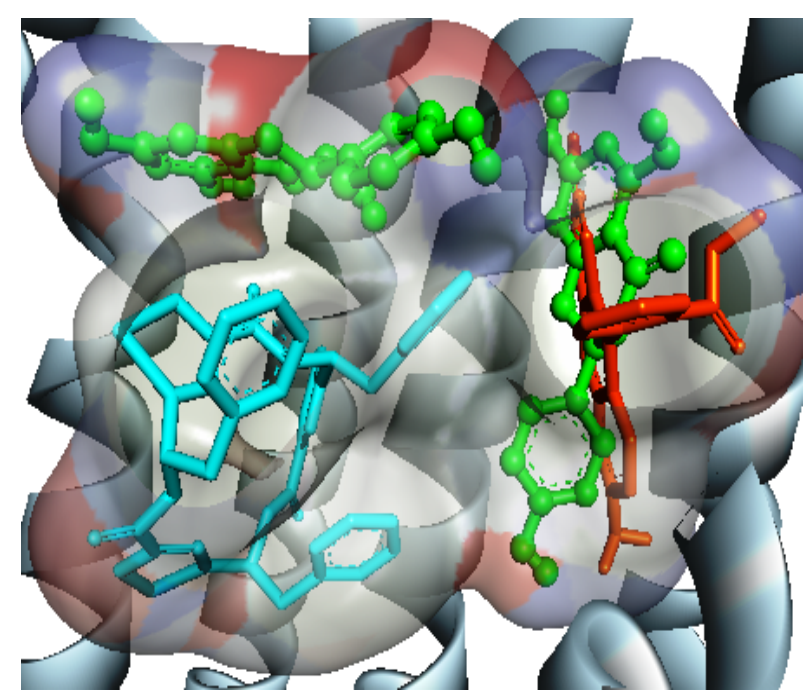

Figure 1. The Docking Pattern of Compounds (1-2) Shown by Ball And Sticks Green Colour, Cocrystallized Ligand (Cyan Colour Sticks) while the Rhodamine is Shown by Sticks Red Colour Against the P-glycoprotein from Mice

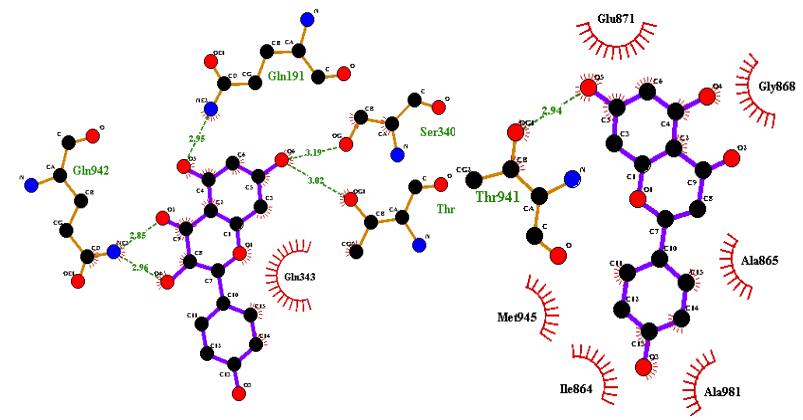

Figure 2. The 2-D Interactions Representation of Compounds 1(left) and 2(right) against P-glycoprotein. In the above Figure Green Dotted Lines Represent Hydrogen Bonding with Distance In Angstrom while Red Half-moon Represents the Hydrophobic Interactions

There are two docking softwares i.e Autodock Vina (Trott and Olson, 2010) and i-GEMDOCKv 2.1 (Hsu et al., 2011) was used for docking studies. Method optimizations of docking softwares were carried out through cocrystallized ligand of P-gp. The Autodock Vina was operated through PyRex tools (Praveen and Yellamma, 2014). Moreover all the default parameters were used for docking through Autodock Vina. Another docking was carried out through i-GEMDOCKv2.1 software. Similarly default procedure was used for docking (Rauf et al., 2015a; Rauf et al., 2015b; Rauf et al., 2015c). The docking analysis was carried out through LIGPLOT+ version v.1.4.5 (Wallace et al., 1995) and Discovery studio visualizer softwares (Visualizer, 2005).

\section{Results and Discussion}

Reversal of Multidrug Resistance in Mouse Lymphoma cells

The effect of multidrug resistance in mouse lymphoma cells is displayed in Table 1. The crude extract showed promising MDR effect, while compounds 1 and 2 was found less active. The fluorescence activity ratio (FAR) value was used to evaluate the $A B C B I$ transporter modulating potential. Among the tested compounds when the compounds were screen in various concentration at $4 \mu \mathrm{g} / \mathrm{ml}$ (Table 1). The values of SSC (side scatter count) and FSC (forward scatter count) were increased in the flowcytometry which showed that the extract and compounds $(1,2)$ had membrane effect and the granulation of cytoplasm was increased. The FAR values obtained by using extract and its compound $(1,2)$ were different, indicating that extract is very effective MDR modulator, and compounds 1 and 2 had no significant effect in a short time experiment. Verapamil, which is a calcium channel blocker and chemosensitizer, was used as a positive control. On MDR mouse lymphoma cells the extract was screen in two concentrations $(4 \mu \mathrm{g} / \mathrm{ml})$. The extract was strong modulators of the efflux-pump activity (FAR 64.02, $4 \mu \mathrm{g} / \mathrm{ml}$ ) while compounds 1 and 2 (FAR 1.58 and 1.79 at $4 \mu \mathrm{g} / \mathrm{ml}$ ) (Table 1).

Computer based drug designing has vital role in predicting the inhibiting potency of new compounds 
against the targeted receptors. Molecular docking studies, was performed to recognize the inhibiting potential of selected flavonoids ( 1 and 2 ) against P-gp from mice. This study co-relates the in-vitro results with in-silico studies. The selected flavonoids and standard Rhodamine were docked into the crystal structure of P-gp. Our docking studies reveal that these compounds give satisfactory docking result on the both docking softwares. As it is cleared from the Figure 1 these compounds not exactly bind in the region where already co-crystallized ligand is present. Generally docking study predicts that, if a compound give lesser interaction energy then that compound has higher activity. Our result of the compounds interaction energies are near to the Rhodamine (Table 1). Hence we can conclude that there are certain structural features of selected flavonoids which are responsible for the inhibitory activities of P-gp from mice.

The docking interactions of the compound 1 (Figure 2) revealed that, it forms a total of five hydrogen bonding interactions. Including them two hydrogen bond are observed from the Gln942 with a distance of $2.85 \AA$ and $2.96 \AA$ while other three hydrogen bond are shown by the residues Gln191, Thr195 and Ser340 with a distance of $2.95 \AA, 3.02 \AA$ and $3.19 \AA$ respectively. Only one hydrophobic contact is observed from the residue Gln343.

The compound 2 (Figure 2) binding to the P-gp is quite weak, because it form only one hydrogen bond interaction with Thr941 with a distance of $2.94 \AA$ while six hydrophobic contacts have been observed from the residues such as Ile864, Ala865, Gly868, Glu871, Meth945 and Ala981.

\section{References}

Ahmad NS, Waheed A, Farman M, et al (2010). Analgesic and anti-inflammatory effects of Pistacia integerrima extracts in mice. J Ethnopharmacol, 129, 250-3.

Assaraf YG (2006). The role of multidrug resistance efflux transporters in antifolate resistance and folate homeostasis. Drug Resistance Updates, 9, 227-46.

Berman HM, Westbrook J, Feng Z, et al (2000). The protein data bank. Nucleic Acids Res, 28, 235-42.

Bihorel S, Camenisch G, Lemaire M, et al (2007). Modulation of the brain distribution of imatinib and its metabolites in mice by valspodar, zosuquidar and elacridar. Pharmaceutical Res, 24, 1720-8.

Brinkhuis M, Izquierdo MA, Baak J, et al (2002). Expression of multidrug resistance-associated markers, their relation to quantitative pathologic tumour characteristics and prognosis in advanced ovarian cancer. Analytical Cellular Pathol, 24, 17-23.

Cole S, Bhardwaj G, Gerlach J, et al (1992). Overexpression of a transporter gene in a multidrug-resistant human lung cancer cell line. Science-New York Then Washington, 258, 1650-.

Cole SP, Deeley RG (2006). Transport of glutathione and glutathione conjugates by MRP1. Trends Pharmacological Sci, 27, 438-46.

Cornwell MM, Pastan I, Gottesman MM (1987). Certain calcium channel blockers bind specifically to multidrug-resistant human KB carcinoma membrane vesicles and inhibit drug binding to P-glycoprotein. J Biological Chem, 262, 2166-70.

Damiani D, Tiribelli M, Calistri E, et al (2006). The prognostic value of $\mathrm{P}$-glycoprotein $(\mathrm{ABCB})$ and breast cancer resistance protein (ABCG2) in adults with de novo acute myeloid leukemia with normal karyotype. Haematologica, 91, 825-8. Diestra JE, Condom E, Del Muro XG, et al (2003). Expression of multidrug resistance proteins P-glycoprotein, multidrug resistance protein 1 , breast cancer resistance protein and lung resistance related protein in locally advanced bladder cancer treated with neoadjuvant chemotherapy: biological and clinical implications. J Urol, 170, 1383-7.

Doyle LA, Yang W, Abruzzo LV, et al (1998). A multidrug resistance transporter from human MCF-7 breast cancer cells. Proceedings National Acad Sciences, 95, 15665-70.

Germann UA, Pastan I, Gottesman MM (1993). P-glycoproteins: mediators of multidrug resistance. Seminars Cell Biol, $\mathbf{4}$, 63-76.

Gottesman MM, Ambudkar SV (2001). Overview: ABC transporters and human disease. J Bioenergetics Biomembranes, 33, 453-8.

Gottesman MM, Fojo T, Bates SE (2002). Multidrug resistance in cancer: role of ATP-dependent transporters. Nature Rev Cancer, 2, 48-58.

Guex N, Peitsch MC (1997). SWISS-MODEL and the Swiss-Pdb Viewer: an environment for comparative protein modeling. Electrophoresis, 18, 2714-23.

Hanwell MD, Curtis DE, Lonie DC, et al (2012). Avogadro: An advanced semantic chemical editor, visualization, and analysis platform. J. Cheminformatics, $\mathbf{4}, 17$.

He S-M, Li R, R Kanwar J, et al (2011). Structural and functional properties of human multidrug resistance protein 1 (MRP1/ ABCC1). Current medicinal chemistry, 18, 439-81.

Hsu KC, Chen YF, Lin SR, et al (2011). iGEMDOCK: a graphical environment of enhancing GEMDOCK using pharmacological interactions and post-screening analysis. BMC Bioinformatics, 12, 33 .

Ismail M, Muhammad N, Mohani N, et al (2011).Pharmacognostic and phytochemical investigation of the stem bark of Pistacia integerrima Stew ex Brandis. J Med Plants Res, 5, 3891-5.

Jara GE, Vera DMA, Pierini AB (2013). Binding of modulators to mouse and human multidrug resistance P-glycoprotein. A computational study. J Molecular Graphics Modelling, 46, $10-21$.

Jones P, George A (2004). The ABC transporter structure and mechanism: perspectives on recent research. Cellular Molecular Life Sciences CMLS, 61, 682-99.

Larkin A, O'Driscoll L, Kennedy S, et al (2004). Investigation of MRP-1 protein and MDR-1 P-glycoprotein expression in invasive breast cancer: A prognostic study. Int J Cancer, 112, 286-94.

Leonard GD, Fojo T, Bates SE (2003). The role of ABC transporters in clinical practice. Oncologist, 8, 411-24.

Li X-Q, Li J, Shi S-B, et al (2008). Expression of MRP1, BCRP, LRP and ERCC1 as prognostic factors in non-small cell lung cancer patients receiving postoperative cisplatin-based chemotherapy. Int J Biological Markers, 24, 230-7.

Li Y, Yuan H, Yang K, et al (2010). The structure and functions of P-glycoprotein. Current Med Chem, 17, 786-800.

Li Z, Wan H, Shi Y, et al (2004). Personal experience with four kinds of chemical structure drawing software: review on chemdraw, chemwindow, ISIS/Draw, and chemsketch. $J$ Chem Informat Computer Sci, 44, 1886-90.

Lopez D, Martinez-Luis S (2014). Marine natural products with P-glycoprotein inhibitor properties. Marine drugs, 12, 525-46.

Praveen K, Yellamma K (2014). Insilco identification of suitable acetuylcholinesterase inhibitors from morinda citrifolia linn. with reference to alzheimer's disease. Int J Pharm Sci Res, 5, 5474 .

Rauf A, Khan R, Raza M, et al (2015a). Suppression of inflammatory response by chrysin, a flavone isolated from 
Potentilla evestita Th. Wolf. In silico predictive study on its mechanistic effect. Fitoterapia, 103, 129-35.

Rauf A, Saleem M, Uddin G, et al (2015b). Phosphodiesterase-1 inhibitory activity of two flavonoids isolated from pistacia integerrima JL stewart Galls. Evidence-Based Complem Altern Med, 2015.

Rauf A, Uddin G, Khan H, et al (2015c). Anti-tumour-promoting and thermal-induced protein denaturation inhibitory activities of $\beta$-sitosterol and lupeol isolated from Diospyros lotus L. Natural Product Res, 1-3.

Robey RW, Polgar O, Deeken J, et al (2007). ABCG2: determining its relevance in clinical drug resistance. Cancer Metastasis Rev, 26, 39-57.

Sakamoto A, Matsumaru T, Yamamura N, et al (2013). Quantitative expression of human drug transporter proteins in lung tissues: analysis of regional, gender, and interindividual differences by liquid chromatographytandem mass spectrometry. J Pharmaceutical Sci, 102, 3395-406.

Sarkadi B, Homolya L, Szakacs G, et al (2006). Human multidrug resistance $\mathrm{ABCB}$ and $\mathrm{ABCG}$ transporters: participation in a chemoimmunity defense system. Physiological Rev, $\mathbf{8 6}$, 1179-236.

Szabo D, Molnar J (1997). The role of stereoselectivity of chemosensitizers in the reversal of multidrug resistance of mouse lymphoma cells. Anticancer Res, 18, 3039-44.

Szakacs G, Paterson JK, Ludwig JA, et al (2006). Targeting multidrug resistance in cancer. Nature Reviews Drug Discovery, 5, 219-34.

Trott O, Olson AJ (2010). Autodock vina: improving the speed and accuracy of docking with a new scoring function, efficient optimization, and multithreading.J Comput Chem, 31, 455-61.

Uddin G, Rauf A, Al-Othman AM, et al (2012a). Pistagremic acid, a glucosidase inhibitor from Pistacia integerrima. Fitoterapia, 83, 1648-52.

Uddin G, Rauf A,Arfan M, et al (2012b). Pistagremic acid a new leishmanicidal triterpene isolated from Pistacia integerrima Stewart. J Enzyme Inhibit Med Chem, 27, 646-8.

Uddin G, Rauf A, Rehman T, et al (2011). Phytochemical screening of Pistacia chinensis var. integerrima. Middle-East J Sci Res, 7, 707-11.

Visualizer DS (2005). Accelrys software inc. Discovery Studio Visualizer, 2.

Wallace AC, Laskowski RA, Thornton JM (1995). LIGPLOT: a program to generate schematic diagrams of protein-ligand interactions. Protein Rngineer, 8, 127-34. 